\title{
Fuzzy Clustering of Stability Diagrams for Structural Health Monitoring
}

E. Peter Carden ${ }^{1}$ and James M.W. Brownjohn ${ }^{1}$

${ }^{1}$ Dept. of Civil and Structural Engineering, University of Sheffield, Sir Frederick Mappin Building, Sheffield S1 3JD, UK

\begin{abstract}
A primary challenge to implementing structural health monitoring techniques on civil infrastructure is the differentiation of effects of environmental variables on the behaviour of structures from other causes of structural change. Data from the Z24 Bridge recorded over the course of nearly a year are analysed in this paper. Covariance-driven Stochastic Subspace Identification is applied to the data and a Fuzzy Clustering Algorithm is used to extract parameters indicative of the bridge's state. The main benefit of this approach is the lack of need for mode shape information and thus it's applicability to structures monitored with spatially sparse sensor grids. The method is shown to provide very encouraging results in separating the response data from the Z24 Bridge in normal and damaged states in varying environmental conditions, and the procedure is then applied to a second data set obtained from monitoring a tall building over several years of its early life in order to identify gradual or sudden structural changes.
\end{abstract}

\section{Introduction}

Throughout the civil, mechanical and aerospace engineering communities there is great interest and benefit to be gained in monitoring a structure to detect damage or monitor inevitable degradation. The development of such techniques allows a shift from schedulebased to condition-based maintenance strategies with consequent economic benefits. This paper is focused on applications to civil infrastructure and in particular to the extraction of characteristic parameters of a structure's behaviour from ambient response histories 
and to the detection of an extraordinary change in a structure's behaviour. This is commonly referred to as level 1 Structural Health Monitoring (SHM). Level 1 is limited to the indication of a change in a structure's behaviour and does not provide information on the location and severity of change or a prognosis for the structure.

There have been many methods published in the literature which attempt to identify damage in a structure, see Doebling et al. (1996), Sohn et al. (2003) and Carden et al. (2004) for reviews of these. When remotely monitoring structures for long periods of time it is economically beneficial to use as small a number of sensors as possible. However, when using a limited number of sensors (as few as one or two), only natural frequencies and damping estimates may be extracted from the response data. Mode shapes cannot be extracted reliably from spatially sparse information and therefore algorithms, which utilise mode shapes and derivatives of mode shapes, may not be used. This paper focuses on extraction of parameters indicative of a structure's state when mode shape information is not available.

A major problem encountered when transferring SHM paradigms from the laboratory to full scale civil structures in the field is the effects of environmental variables, in particular temperature, on the modal parameters of the structures. This is a difficulty not encountered with rotating machinery (Farrar and Doebling, 1999), which is probably the most mature and successful application area of SHM.

For example, Cornwall et al. (1999) examined two days of data from the single span Alamosa Canyon Bridge. They found up to $6 \%$ variability in the measured modal parameters over a 24 hour period which they found to be correlated with temperature measurements. They concluded that a required prerequisite for a remote bridge health 
monitoring system is a thorough understanding of the variability of dynamic properties caused by the changing environmental and operational conditions.

An excellent dataset that can be used to examine the feasibility of separating the effects of environmental variables from the effects of structural changes is that obtained using the Swiss Z24 Bridge (Peeters and De Roeck, 2001). The Z24 Bridge was a post tensioned concrete box girder bridge with a main span of $30 \mathrm{~m}$ and two side spans of 14 m. It was monitored from 11 November 1997 until 11 September 1998 as part of the European SIMCES project and the dataset maybe downloaded from the SAMCO database (www.samco.org). Several damage scenarios were inflicted at the end of the monitoring period including settlement of a pier, rotation of a foundation, and induced failure of a concrete hinge, anchor heads and post tensioning wires. Details of these damage tests may be found in Kramer et al. (1999).

Peeters and De Roeck (2001) examined the data recorded from Z24 Bridge in detail. An automated modal analysis procedure was implemented based on analysing the stability diagrams produced from applying stochastic subspace identification (SSI) to each hourly recorded 10 minute data set. Natural frequencies, damping and mode shapes were extracted. Subsequently, Autoregressive eXogeneous (ARX) models were fitted to the natural frequencies of the first four modes. Damage events inflicted on the bridge at the end of the year were successfully distinguished from changes due to environmental variables during the year. Of note is that data recorded when the asphalt layer froze and the modal parameters displayed signs of significant stiffening of the bridge were excluded from the training and testing datasets.

This paper also utilises SSI for automatic modal parameter extraction but differs from Peeters and De Roeck (2001) in that the sensor grid is assumed to be too sparse to 
provide meaningful mode shape estimates. This is attempted so as to assess the possibility of using a limited number of sensors for continuous remote monitoring of civil structures.

In the next section a short review of SSI theory is provided to give the necessary background for the discussion of extracting modal parameters automatically from stability diagrams. Difficulties are encountered when mode shape information is not available and this leads the authors to utilise fuzzy clustering techniques to extract indicative measures of natural frequencies and damping. The theory behind fuzzy clustering is briefly described and its application to the data from the Z24 Bridge is demonstrated. The technique is shown to identify successfully damage inflicted on the Z24 Bridge. The paradigm is then applied to the Republic Plaza Office Building in Singapore. The shifts in several modes of the building over a seven year period are clearly captured. The main contribution of this approach is that mode shape estimates are not needed, enabling its use with low-cost monitoring systems using only a few sensors.

\section{Stochastic Subspace Identification}

Mathematically, structural systems may be recast from continuous second order differential equations governing their behaviour into discrete state space equations. SSI is an efficient method for identifying the state space matrices from measured response data. The natural frequencies, damping parameters and mode shapes may then be extracted from these state space matrices. Major advantages of taking this approach are that it is quick and non-iterative and therefore does not suffer from convergence problems. Fundamental proofs and discussions of numerical stability are described in Van Overschee and De Moor (1996), while a succinct, though thorough, step-by-step procedure with an example application is provided in Peeters and De Roeck (1999). 
There are various flavours of SSI available and here we use reference-based covariancedriven stochastic realisation as described in detail in Peeters and De Roeck (1999) and utilised in Peeters and De Roeck (2001). The advantage of using covariance-driven realisation as opposed to data-driven subspace projection is that the data reduction (which is effectively a major noise filtering step) is far less memory intensive. The data-driven subspace projection utilises a QR decomposition of the data Hankel matrix and for large data sets this becomes very demanding on computer memory. The covariance-driven realisation requires the formation of the Toeplitz matrix (Eq. 1) of the covariance of the measured output and is far less demanding on memory.

$$
T_{1 \mid i}=\left(\begin{array}{ccc}
\Lambda_{i} & \Lambda_{i-1} & \Lambda_{1} \\
\Lambda_{i+1} & \Lambda_{i} & \Lambda_{2} \\
& & \\
\Lambda_{2 i-1} & \Lambda_{2 i-2} & \Lambda_{i}
\end{array}\right)
$$

The subscript on the Toeplitz matrix of covariances, $T$, refers to the subscripts of the first and last entries of the first row of the matrix. The subscript on the covariance matrix $\Lambda$ between all measured outputs and the analyst-chosen reference outputs refers to the covariance lag. $T_{1 \mid i}$ is singular value decomposed according to Eq. 2 and this implicitly implies the choosing of the order of the system, $n$.

$$
T_{1 \mid i}=U S V^{T}=\left(\begin{array}{ll}
U_{1} & U_{2}
\end{array}\right)\left(\begin{array}{cc}
S_{1} & 0 \\
0 & S_{2}
\end{array}\right)\left(\begin{array}{c}
V_{1}^{T} \\
V_{2}^{T}
\end{array}\right) \rightarrow U_{1} S_{1} V_{2}^{T}
$$

In theory the number of non-zero singular values will equal the order of the system, $n$. In Eq. 2 the singular value matrix is broken into $S_{1}$ and $S_{2}$. With perfect numerical accuracy and absolutely clean data, $\mathrm{S}_{1}$ would comprise a diagonal of $n$ non-zero entries and $\mathrm{S}_{2}$ would be null matrix. In such a case, $T_{1 \mid i}$ would decompose to $U_{1} S_{1} V_{1}^{T}$. However, in practice this will not happen. On rare occasions, with very good quality data and with some luck, there is a dramatic drop in the singular values which marks the order of the 
system. All too often this step down in magnitude of value is replaced by a gradual reduction and it is up to the analyst's skill and experience to choose the correct cut off order. Once an order is chosen, the dynamic state space matrix may be calculated, by utilising the singular values. The eigenvalues of this dynamic matrix are the poles of the system and thus yield the natural frequencies and damping estimates while the eigenvectors may be converted to the mode shape vectors. Details may be found in Peeters and De Roeck (1999).

The construction of a stability diagram has proven invaluable in helping the analyst to choose the correct order of the system and distinguish between true modal parameters and mathematical chimera. The stability diagram is formed by choosing a range of values for $n$ and extracting the modal parameters from the dynamic matrix for each of these values. The analyst defines rules by which modal parameters may be considered stable between two different assumed orders. Figure 1 is an example of a stability diagram from data recorded on the Z24 Bridge. The rules used in this case to define a pole as being stable were:

- variation in the natural frequency of less than $1 \%$

- variation in damping of less than $5 \%$

- Modal Assurance Criterion (MAC) value of at least 0.98 .

Figure 1 shows four clear columns with identified stable poles from a low order consistently up to the highest order. These columns are at about $3.8 \mathrm{~Hz}, 4.6 \mathrm{~Hz}, 9.8 \mathrm{~Hz}$ and $10.4 \mathrm{~Hz}$ and are the first four modes of the bridge. This diagram is quite easy to interpret but Figure 2 shows another stability diagram from the Z24 Bridge Dataset recorded at a different time. This diagram is not so easy to interpret and without access to further recorded data it is unlikely that an analyst would be able to pick out the correct four modes with confidence. 
Peeters and De Roeck (2001) described a procedure for automatic selection of the modal parameters by choosing only those poles that were five times stable. They stated that this criterion filtered out accidentally stable poles from stable poles of physical modes. Using this method they were able to extract the first four modes automatically with great success. In this paper, concentration is made on not utilising information of the mode shapes. In doing so, only the first two rules in choosing stable poles stated above are applicable.

The results of applying these two rules and using a ' $\mathrm{k}$ times stable' criterion of $\mathrm{k}=15$ is shown in Figure 3 for the complete dataset recorded from eight accelerometers. A high value of $\mathrm{k}$ was used as the results with a lower $\mathrm{k}$ were poor. The real modes can be seen to stand out as thick bands. There are two modes visible in Figure 3a beginning at about 4.0 $\mathrm{Hz}$ and $5.1 \mathrm{~Hz}$, and two modes visible in Figure $3 \mathrm{~b}$ beginning at about $10.2 \mathrm{~Hz}$ and 10.7 Hz. There are clear increases in value of the natural frequencies with the most prolonged increase from approximately hour 1800 to hour 2300 . These increases are due to freezing of the asphalt and are investigated more fully in Peeters and De Roeck (2001). The gaps in Figure 3 correspond to periods when the monitoring system was not operating.

The number of mathematical poles in Figure 3 is large and makes automatic picking of the real mode estimates difficult. Clearly the automatic selection has not been as successful as when including the mode shape information in Peeters and De Roeck (2001). Requiring a higher times stable criterion results in a less cluttered diagram but also in erroneously rejecting real stable poles and therefore not identifying the modes. 
The authors attempted to track the modes across Figures $3 \mathrm{a} \& 3 \mathrm{~b}$ using a criterion that the next pole in time would be the closest pole in a Euclidean sense (a nearest neighbour approach). Each pole contained information on frequency and damping. The mode shape information was purposefully neglected. This criterion was augmented by a maximum allowable Euclidean distance to avoid choosing a mathematical pole when a real mode was weakly excited and not identified. This approach had limited success. The trends of the first two modes were reasonably extracted though there was uncertainty that each pole selected was a real pole as opposed to a mathematical pole that happened to be closest to the previously selected pole. The trends of the second two poles could not be extracted successfully. The trends extracted deviated substantially from the thick bands seen in Figure 3a. Their closeness and the greater number of mathematical poles in this frequency region led to the failure. With mode shape information it would be possible to use a criterion such as the MAC value to track modes across time in Figure 3 and be far more successful.

After trial and error with various combinations of values of number of covariance estimates (parameter $i$ in Eq. 1), reference channels and stability criteria, it was concluded by the authors that this approach was failing. Without mode shape information it was found to be impossible to track the progression of the modes across Figure 3 with reliability. It was especially error prone for modes 3 and 4 during the freezing periods.

Due to these difficulties, fuzzy clustering of the identified poles was investigated and is described and demonstrated in the next section.

\section{Fuzzy Clustering of the Identified Poles}

Since about 1950 a vast array of literature examining statistical clustering algorithms has developed. This is largely due to the wide spread of areas to which clustering has been 
applied, including the natural sciences (alpha taxonomy being an obvious example), through the social sciences, economics and engineering (Hartigan 1975). In this paper we will focus on the use of Fuzzy C-Means clustering (FCM) which was originally developed by Bezdek (1981).

FCM is essentially concerned with the separation of a dataset into overlapping subsets. The number of subsets is specified by the analyst $a$ priori and is typically denoted by $\mathrm{C}$ (which gives rise to its name). A brief review of the ideas behind FCM is given below; further mathematical and implementation details are available in Bezdek (1981). The implementation of FCM used throughout this paper is that provided in the Matlab Fuzzy Logic Toolbox (The Mathworks, Inc., 2004).

Essential to the understanding of fuzzy clustering is the concept of the membership matrix $\boldsymbol{U}$. Each element of $\boldsymbol{U}, u_{i k}$, denotes the membership of element $x_{k}$ of a dataset to cluster $i$. Each data point $x_{k}$ has memberships that sum to exactly 1 . The algorithm begins by assuming a set of cluster centres denoted by $v_{i}$ and minimising the objective function in Eq. 3.

$$
J=\sum_{i=1}^{C} \sum_{k=1}^{N}\left(u_{i k}\right)^{m}\left(x_{k}-v_{i}\right)^{T}\left(x_{k}-v_{i}\right)
$$

$N$ is the number of members of the dataset. The exponent $m$ determines the fuzziness of the clusters or to what extent data points may belong to multiple clusters. A typical value chosen is two and this value is used throughout this paper. The algorithm proceeds by iteratively updating the cluster centres and the membership function to find a minimum.

Scionti and Lanslots (2005) used FCM techniques to process automatically stability diagrams and choose real modes. They first plotted frequency against damping of each identified pole and noticed a clear grouping of poles associated with real modes. The groupings were elongated in the vertical axis due to the greater variability of the 
estimated damping parameter. Figure 4 shows the stable poles from Figure 1 in the frequency bands of $3.5 \mathrm{~Hz}$ to $6 \mathrm{~Hz}$ and $9.3 \mathrm{~Hz}$ to $12.2 \mathrm{~Hz}$ plotted in such a way. The elongated groupings are clearly seen. Traditional FCM (as described with Eq. 3) forms hyperspherical clusters. The clusters in Figure 4 are clearly not spherical in form, and so one is proverbially attempting to fit a square peg into a round hole. Scionti and Lanslots (2005) found greater success by using the more advanced Gustafson-Kessel FCM algorithm which allows the formation of hyperellipsoidal clusters. This is achieved by using the covariance matrix of the data points to modify Eq. 3. Data points that have an equal distance metric in the Gustafson-Kessel FCM algorithm form an ellipsoid when measured by the Euclidean metric.

In this paper we propose not to use the clusters formed by the frequency and damping parameter estimates but rather those formed by the real and imaginary parts of the discrete poles as plotted on the Z-plane. Eq. 4 shows the relationship between the pair of complex conjugate poles, $\lambda$, and the undamped natural frequency, $\omega_{\mathrm{n}}$, and damping parameter, $\zeta$.

$$
\lambda, \lambda^{*}=e^{\Delta\left(-\zeta \omega_{n} \pm \omega_{n} \sqrt{\zeta^{2}-1}\right)}
$$

$\Delta$ is the time difference between measurements. The same identified poles as seen in Figure 4 are shown in Figure 5 on the Z-plane. The clusters seen are clearly far less ellipsoidal and this allows the use of the simpler FCM algorithm. It also removes the computational effort which is required to calculate the frequency and damping estimates from the poles, though this is minor.

Figure 5 depicts results from analysing just one recorded dataset of 10 minute duration. It is not clear just which groupings represent real modes and which do not. Figure 6 depicts the results of analysing a day of recordings (that is 24 datasets of 10 minute duration) and 
is zoomed in on the cluster formed by the first mode, with an X marking the centre of the cluster as determined by the FCM algorithm. Dense clusters form around all the real modes and the FCM algorithm is successfully able to pick these out from the surrounding less dense cloud of mathematical poles.

The purpose of this paper is not automatic pole selection but rather the condensation and extraction of parameters from datasets which are indicative of the state of the structure. If the structure were to change its state, then its modes would also shift. If the modes shift then the centres of the clusters would also shift. Therefore the tracking of the centres of the clusters formed would provide an indicator of changes in the structure's state as reflected in its dynamic characteristics. The centres of the clusters are found automatically by using the FCM algorithm described above.

This approach to extracting parameters indicative of the structure's state is demonstrated on the Z24 Bridge in the next section.

\section{Application to Structural Health Monitoring}

\subsection{The Z24 Bridge}

Data were recorded every hour from eight accelerometers mounted on the Z24 Bridge over the course of nearly a year with intermittent gaps. Covariance driven SSI was applied to each of these datasets. All stable poles identified using the criteria previously stated for natural frequency and damping estimates were recorded. The mode shape information was purposefully neglected. This results in more mathematical poles passing the $\mathrm{k}$ times stable criteria described earlier and also makes the tracking of modes through time more difficult. The stable poles identified over the course of each day, i.e. 24 sets of poles, were fed to the FCM algorithm in two batches. The first batch contained all poles in the frequency band of $3.5 \mathrm{~Hz}$ to $6 \mathrm{~Hz}$. This band contained two modes of the bridge 
and two cluster centres were identified. The second batch contained all poles in the frequency band of $9.3 \mathrm{~Hz}$ to $12.2 \mathrm{~Hz}$. This band also contained two modes of the bridge but included far more mathematical poles than the first band. Three cluster centres were identified. Two of these clusters were related to the two modes of the bridge. The third cluster was used to capture the large number of mathematical poles in this frequency band.

The cluster centres identified over the entire monitoring period of the bridge are shown in Figure 7. Figure $7 \mathrm{~b}$ depicts the cluster centres formed from the poles in the frequency band of $3.5 \mathrm{~Hz}$ to $6 \mathrm{~Hz}$. Figure $7 \mathrm{a}$ depicts the cluster centres formed from the poles in the frequency band of $9.3 \mathrm{~Hz}$ to $12.2 \mathrm{~Hz}$. The grey dots cluster centres identified before damage was inflicted and the black dots are centres identified after damage was inflicted. The upper left clusters of grey and black in Figure 7a are dominated by mathematical poles. Four other pairs of grey and black clusters are clearly distinguishable in Figures $7 \mathrm{a} \& \mathrm{~b}$ and relate to the first four modes of the bridge. The tail of grey dots coming anticlockwise from the grey dot clusters are related to the periods when the asphalt on the bridge was frozen and apparently contributed significant stiffness to the bridge. This shift in behaviour is also visible in Figure 3 as previously stated.

The clear separation in the groups of grey (healthy state) and black dots (unhealthy state) in Figure 7 is very encouraging towards using this technique as a structural health monitoring paradigm. Of note is that no information regarding the environmental conditions was used, which of course varied over the course of the year of monitoring. This information is available for the Z24 Bridge and further development work is being undertaken by the authors to include this information to enhance the separation of states of the structure. Additionally, robust statistical inference techniques to interpret the groupings seen in Figure 7 in a reliable manner are under investigation. 


\subsection{Republic Plaza Office Tower}

As a proof of concept, the paradigm is applied to data recorded from the $280 \mathrm{~m} 66$ storey Republic Plaza Office Tower in Singapore. A full description of Republic Plaza is given in Teh and Lai (1995). Figure 8 shows a perspective view of the building and Figure 9 shows a typical cross section at lower levels. The tower has a frame-tube structural system with an internal core wall connected to a ring of external columns by horizontal steel-framing system at every floor.

The building symmetry suggests a pair of axes, labelled A and B as shown in Figure 9, for measurement of building vibration. The arrangement of lift shafts and core wall openings up to the highest level suggests that B will be the stiffer direction.

As part of a project to characterize wind loading and dynamic response of tall buildings in Singapore, an instrumentation system was installed on Republic Plaza. Details of the system are given in Brownjohn \& Pan (2001). For the majority of operational time the system recorded data only when triggered by a pre-set level of response. However, for shorter periods the system acquired data continuously. Two of these continuously recorded datasets are examined here.

The first dataset was recorded from 10 January 1997 until 28 January 1997 and shall be referred to as RP1. A total of 3632 frames with 4096 data points each were recorded with a sampling rate of $9.375 \mathrm{~Hz}$. The second dataset was recorded from 30 December 2003 until 8 January 2004 and shall be referred to as RP2. A total of 1490 frames with 4096 data points each were recorded with a sampling rate of $8 \mathrm{~Hz}$. In both sets, responses from two orthogonal accelerometers located near the top of the building are used. 
Covariance driven SSI was applied to all of the frames with both available accelerometer channels used as reference channels. Eighty lines of the estimated covariance were used to build the Hankel matrix of covariances (Eq. 1). Systems with orders of 1 to 80 were extracted. The same criteria used with the Z24 dataset for determining stable poles were also applied to both RP1 and RP2. In this paper, concentration is focused on the first five modes of the building which are all below $0.8 \mathrm{~Hz}$.

The frequencies of the stable poles under $0.8 \mathrm{~Hz}$ determined for each frame are shown in Figure 10a. Frames 1 to 3632 are from RP1 and frames 3633 to 5122 are from RP2. They are plotted together to show the shifts in the first five modes. Figure $10 \mathrm{~b}$ shows a zoom in on the first two modes just below $0.2 \mathrm{~Hz}$, which are indistinguishable from each other in Figure 10a. It is noticeable that there is little shift in the frequencies of these modes between the RP1 and RP2. The third mode is just above $0.5 \mathrm{~Hz}$ and there is a clear shift in its value between RP1 and RP2. Figure 10c shows a zoom in on the fourth and fifth modes which are hidden by spurious modes in Figure 10a. There is a noticeable shift in both modes' frequencies between RP1 and RP2 due to ageing of the structure over the intervening seven year period between measurements.

Figure 10a exhibits a ripple of vertical bands. These are composed of spurious stable poles and coincide with night time and weekends. The excitation of the building is greater during weekday daytime than at night and at weekends due to occupation by workers and operation of machinery such as the lifts. The greater excitation provides a clearer stabilisation diagram and hence the decrease of spurious poles in Figure 10a during the day time periods. This shift in quality of stabilisation diagram provides a challenge to automatic modal analysis and structural health parameter monitoring. The fuzzy clustering based paradigm is tested with this challenging dataset as a proof of concept. 
The poles from periods of four hours were grouped together and fed to the fuzzy clustering algorithm. Due to the different sampling rates, poles from sets of 33 frames in RP1 and from sets of 28 frames in RP2 formed four hour groups. The results from RP1 are examined in detail first before comparison with results from RP2.

The first two modes are very close in frequency value. This represented a real difficulty to the clustering algorithm. The two modes are closely spaced clusters while the other modes are relatively distant clusters. The algorithm consistently grouped the first two modes together in one cluster because of this ill-conditioning in cluster spacing. If desired, two approaches could be used to identify separate clusters for these two modes. Firstly, the responses from two accelerometers could be analysed separately. Due to their orthogonal orientation in line with the axes of the building, only one mode would appear in each analysis. Secondly, the frequency band that the clustering is applied to could be narrowed. This would leave just two clusters and remove the ill-conditioning of very different between cluster spaces. However, the grouping of the first two modes together is not a critical problem for the purposes of this paper and neither method is employed. Rather extracting parameters suitable for long term structural monitoring, which is the main thrust of the paper, is focused on.

The fuzzy algorithm was employed with a range of preset number of clusters. Using five clusters gave best results. One cluster represented the first two modes, three of the clusters represented the remaining three modes and one cluster represented the spurious poles in frequency band between the first two modes and the third mode. Figure 11 shows all the cluster centres extracted from the four hour groups from RP1 plotted on the Zplane. To help with visualisation, a segment of the unit circle and radii at frequencies of $0.2,0.4,0.6$ and $0.8 \mathrm{~Hz}$ are also drawn in Figure 11. 
Six clusters are visually distinguishable in Figure 11 and the fuzzy clustering algorithm was applied to this dataset. The centres of the clusters found are marked by stars in Figure 11 and numbered from one to six. Six clusters were fitted, and not five as were fitted to generate the data, because the fourth and fifth modes were weakly excited at night time and during weekends. This led to the frequency range around these modes being surrounded by many spurious mathematical poles and the fuzzy clustering algorithm identified a cluster nearer to $0.8 \mathrm{~Hz}$ (marked as cluster 6 in Figure 11) based on these spurious poles and not the real poles. A sixth cluster is therefore employed to account for this spurious group.

It is naturally desirable to be able to filter and reject those data in clusters 2 and 6 in Figure 11. This may be achieved by examining the membership and compactness of each cluster, which are reported in Table 1. In this paper, a data point is considered a member of a cluster if its membership factor ( $u_{i k}$ in Eq. 3$)$ is greater than 0.5. If a cluster has a relatively large percentage of the data as members, then there can be confidence that it is a significant cluster. The compactness of a cluster is the mean Euclidean distance between the centre of the cluster and those points which are deemed to be members of that cluster using the criterion for membership just stated. If the compactness of a cluster is relatively low compared to other clusters then it is likely that this cluster is collecting a larger number of outliers or noise. Both clusters 2 and 6 have the lowest values of membership and the highest values of compactness and can therefore be rejected. For the purposes of structural monitoring, clusters $1,3,4$, and 5 can therefore be used to track the performance of the structure and identify shifts and anomalies in the structure's behaviour. 
Figure 12 shows the centres of the clusters extracted from RP2 marked as ' + ' compared with those from RP1 marked as ' $’$ The centres have been rotationally transformed on the Z-plane to account for the difference in sampling frequency between the two data sets.

The centres from RP1 and RP2 overlap substantially in cluster 1 . This cluster is related to modes 1 and 2 and the overlap is consistent with the very small shift in these modes seen in Figure 10b. There is a clear shift in the centres in clusters 3, 4 and 5. The centres from RP2 are shifted clockwise compared to those from RP1 and this is consistent with the shifts in modes 3, 4 and 5 seen in Figures 10a \& 10b.

The paradigm has been able to reduce these two large datasets to sets of four parameters (cluster centres) which are indicative of the structural behaviour. The change in the building's dynamic characteristics occurring over a period of seven years was captured by shifts in three of the parameters. Again, a well founded statistical test of significance of the cluster centres is being investigated by the authors. The separation of effects on a structure caused by long term trends from sudden damage is still an outstanding issue.

\section{Conclusion}

This paper focused on the condensation of monitoring data and the extraction of indicators of a structure's state. The use of automatic pole selection worked well for previous authors when mode shape information was available. When a very limited grid of sensors is installed on a structure, mode shapes may not be extracted from the data. Poles were identified in data recorded over the course of a year from the Swiss Z24 Bridge. The automatic filtering of real poles, related to the state of the structure, from mathematical poles was made more difficult with the lack of mode shape information. Due to this difficulty, a fuzzy clustering algorithm was applied to the identified poles. The centres of the clusters identified were then used as indicators of the state of the Z24 Bridge. Shifts in these indicators were clearly seen when damage was inflicted. The 
paradigm was also applied to the Republic Plaza Office Tower in Singapore. The change in dynamic characteristics of the building over a period of seven years was captured by shifts in the centres of the clusters identified. The results are encouraging but more development is needed to develop robust statistical tests for these indicators and to include information of the environmental conditions under which the data was recorded. The use of modal identification methods that give clearer stability diagrams such as Polymax may also help, although care must be taken with such a method as weakly excited modes will be forced outside the unit circle on the Z-plane and therefore deemed not stable. The investigation of alternative clustering algorithms may also prove fruitful. 


\section{References}

Bezdek JC (1981), Pattern recognition with fuzzy objective function, Plenum Press, New York.

Brownjohn, J.M.W. \& Pan, T.C. (2001), Response of tall buildings to weak long distance earthquakes, Earthquake Engineering and Structural Dynamics, 30,709-729.

Carden, E.P. and Fanning, P. (2004), Vibration Based Condition Monitoring: A Review, Structural Health Monitoring, 3(4), 355-377.

Cornwell, P.J., Farrar, C.R., Doebling, S.W. \& Sohn, H. (1999), Environmental Variability Of Modal Properties, Experimental Techniques, Nov/Dec, 45-48.

Doebling, S.W., Farrar, C.R., Prime, M.B. \& Shevitz, D.W. (1996), Damage Identification and Health Monitoring of Structural and Mechanical Systems from Changes in their Vibration Characteristics: A Literature Review, Los Alamos National Laboratory Technical Report LA-13070-MS.

Farrar, C.R. \& Doebling, S.W. (1999), Damage detection II: field applications to large structures, in: Silva, J.M.M. and Maia, N.M.M. (eds.), Modal Analysis and Testing, Nato Science Series, Kluwer Academic Publishers, Dordrecht, Netherlands.

Hartigan, J.A. (1975 ), Clustering Algorithms, John Wiley \& Sons, New York

Kramer, C., De Smet, C.A.M. \& De Roeck, G. (1999), Z24 Bridge Damage Detection

Tests, in proceedings of the 17th International Modal Analysis Conference (IMAC),

Kissimee, Florida, pp. 1023-1029. 
Peeters, B. \& De Roeck, G., Reference-Based Stochastic Subspace Identification For Output-Only Modal Analysis, Mechanical Systems and Signal Processing (1999) 13(6), $855-878$.

Peeters, B. \& De Roeck, G. (2001), One-year monitoring of the Z24-Bridge: environmental effects versus damage events, Earthquake Engineering and Structural Dynamics, 30, 149-171.

Scionti, M. \& Lanslots, J.P. (2005), Stabilisation diagrams: Pole identification using fuzzy clustering techniques, Advances in Engineering Software, 36, 768-779.

Sohn, H., Farrar, C.R., Hemez, F.M., Shunk, D.D., Stinemates, D.W. \& Nadler, B.R. (2003), A Review of Structural Health Monitoring Literature: 1996-2001, Los Alamos National Laboratory Report, LA-13976-MS.

Teh, H.S. \& Lai H.P. (1995), Structural aspects of the Republic Plaza in proceedings of the Seminar on Tall Buildings - Design and Construction, The Institution of Engineers Singapore.

The MathWorks, Inc. (2004), Fuzzy Logic Toolbox for Matlab, Massachusetts, USA.

Van Overschee, P. \& De Moor, B. (1996), Subspace Identification for Linear Systems, Kluwer Academic Publishers, USA. 


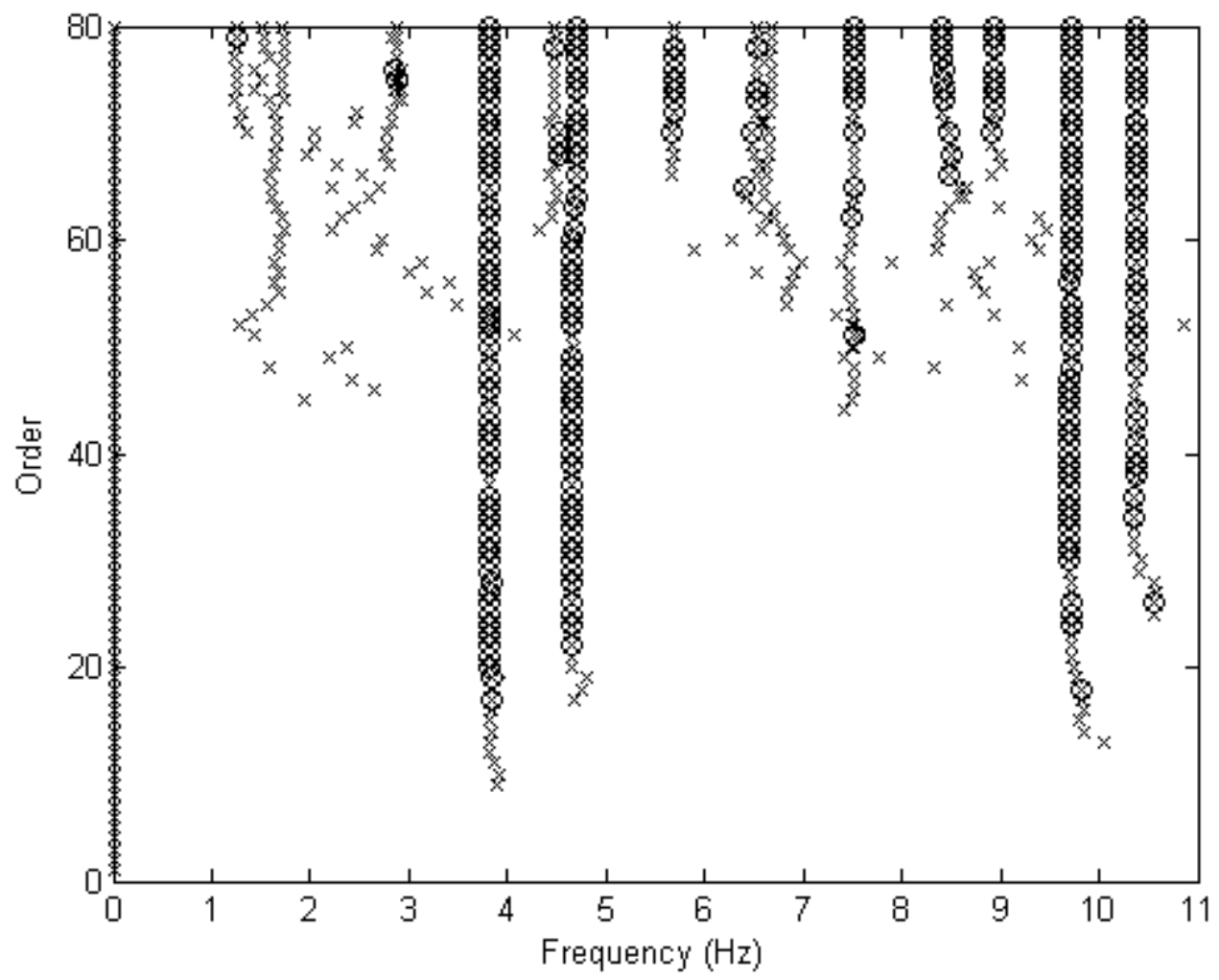

Figure 1. A clear stabilisation diagram from the Z24 Bridge Dataset. ' $x$ ' is an identified unstable pole. 'o' is an identified stable pole. 


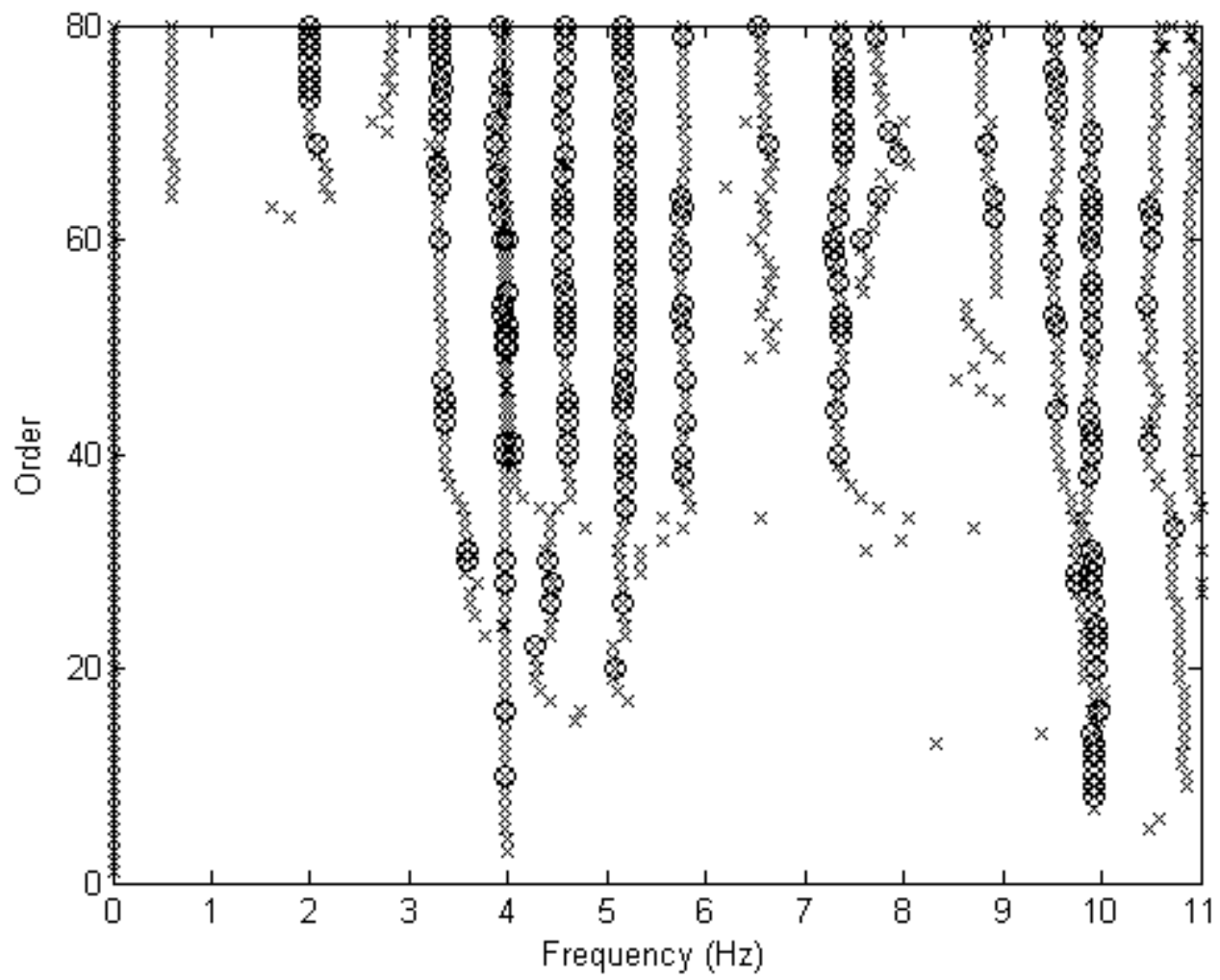

Figure 2. An unclear stabilisation diagram from the Z24 Bridge Dataset. ' $x$ ' is an identified unstable pole. 'o' is an identified stable pole 


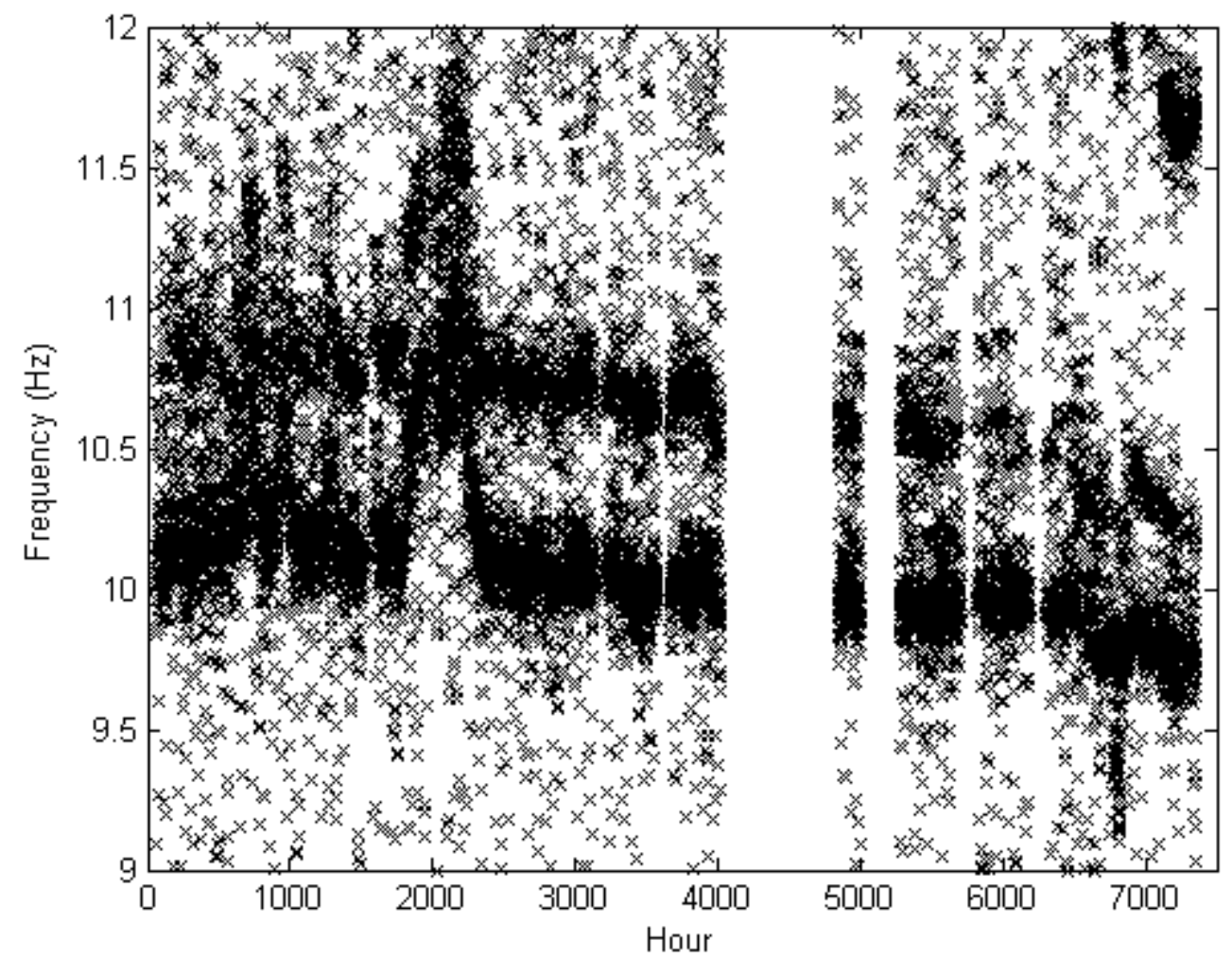

Figure 3a

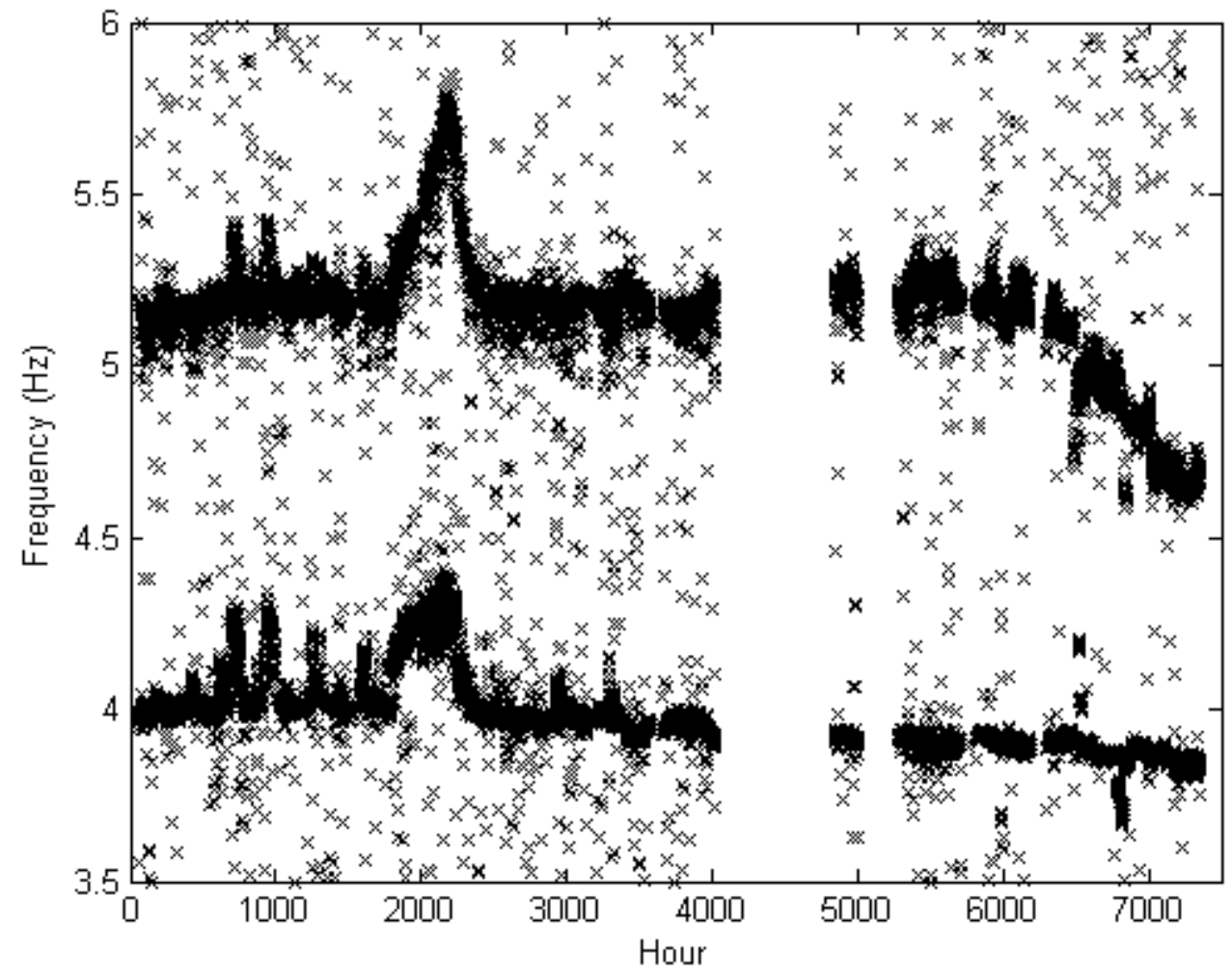

Figure $3 b$

Figure 3. The thick bands across (a) and (b) are the first four modes of the bridge. The random cloud of Xs between these bands is composed of mathematical poles. 


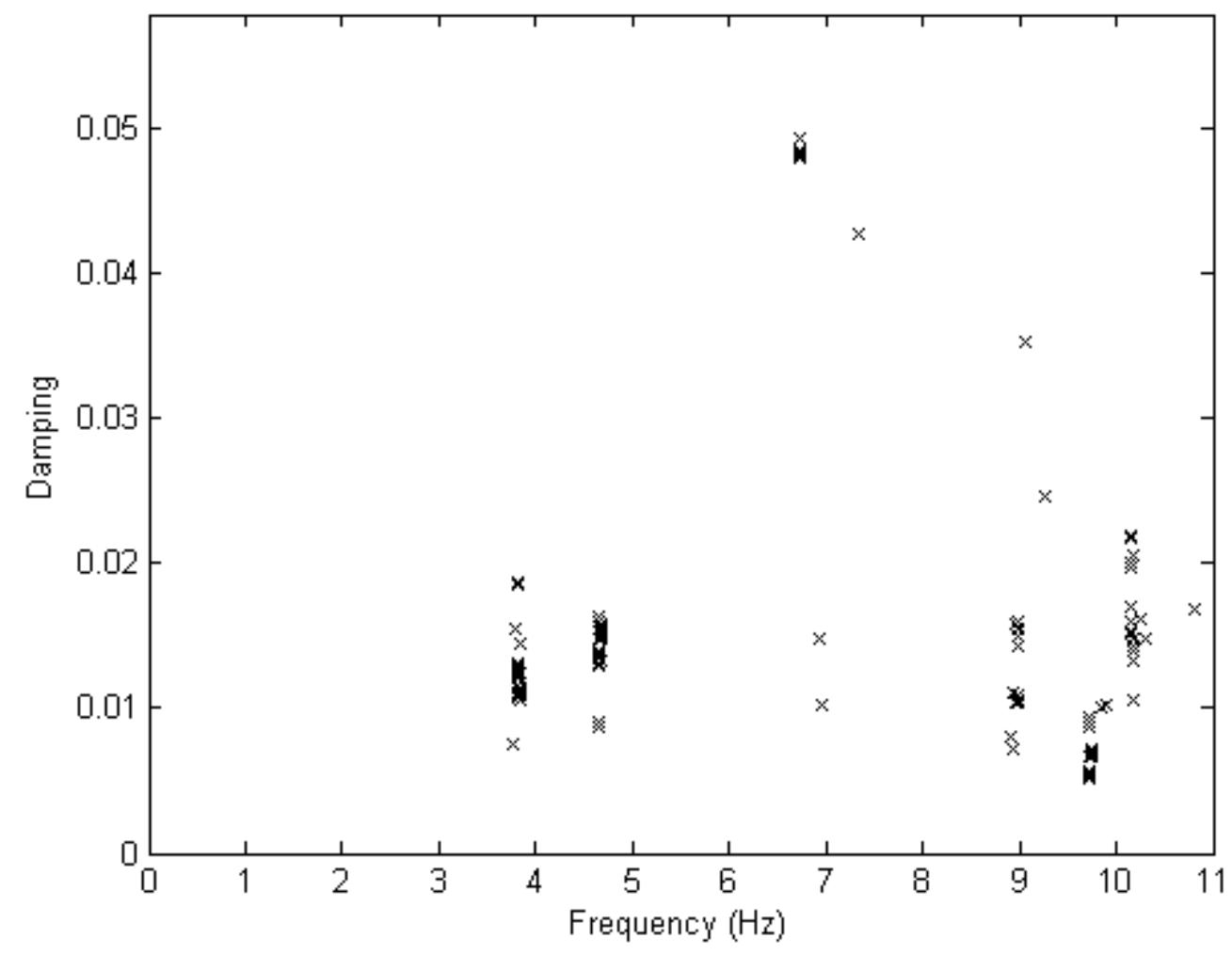

Figure 4 Elongated clusters formed from frequency and damping estimates 


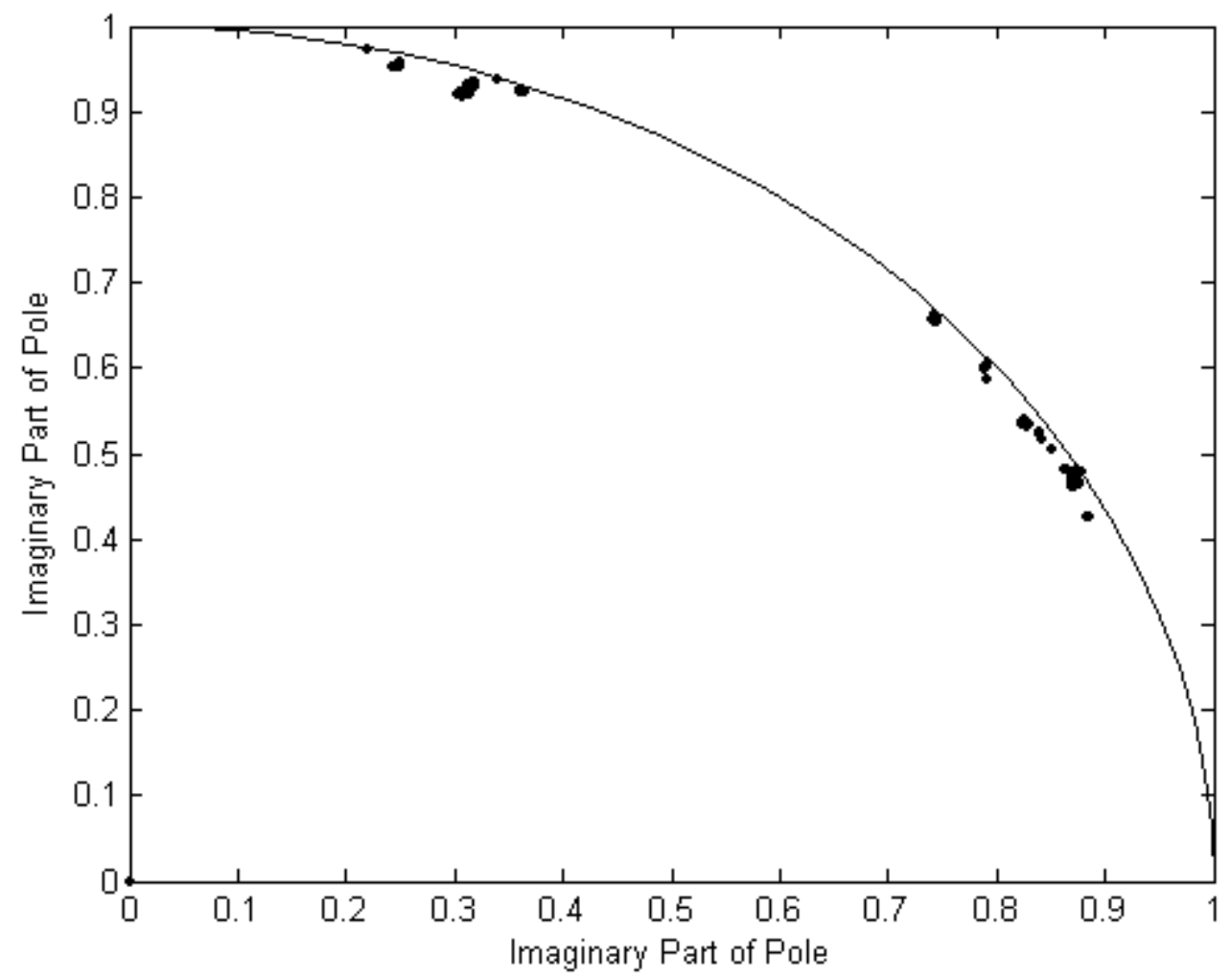

Figure 5. Clustering of poles on the Z-plane. The solid line is the circumference of the unit circle for reference. 


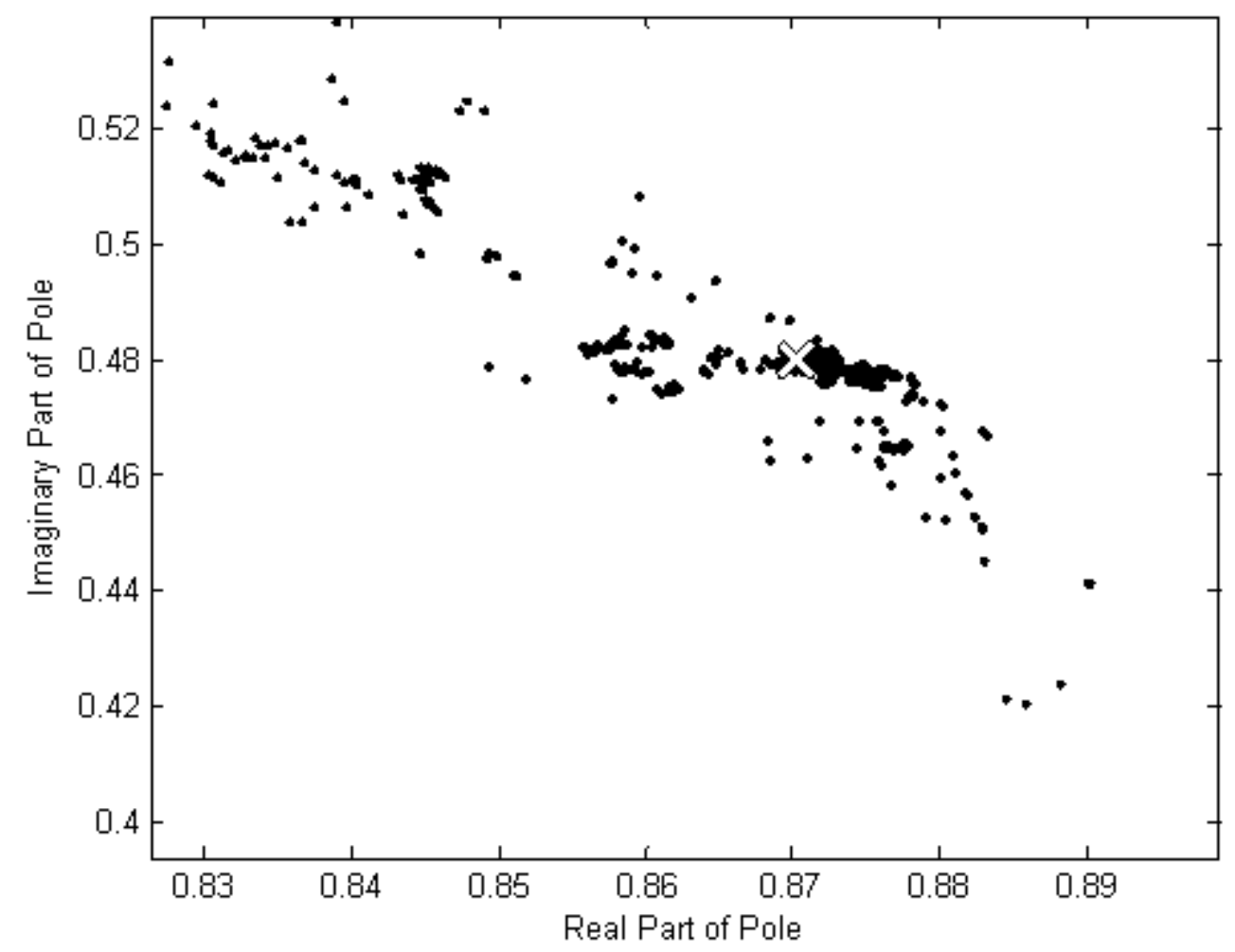

Figure 6. Zoom in of cluster formed by the first mode of the Z24 Bridge data recorded over one day. Gray dots are the Poles. The white ' $\mathrm{X}$ ' is the centre of the cluster found by FCM. 

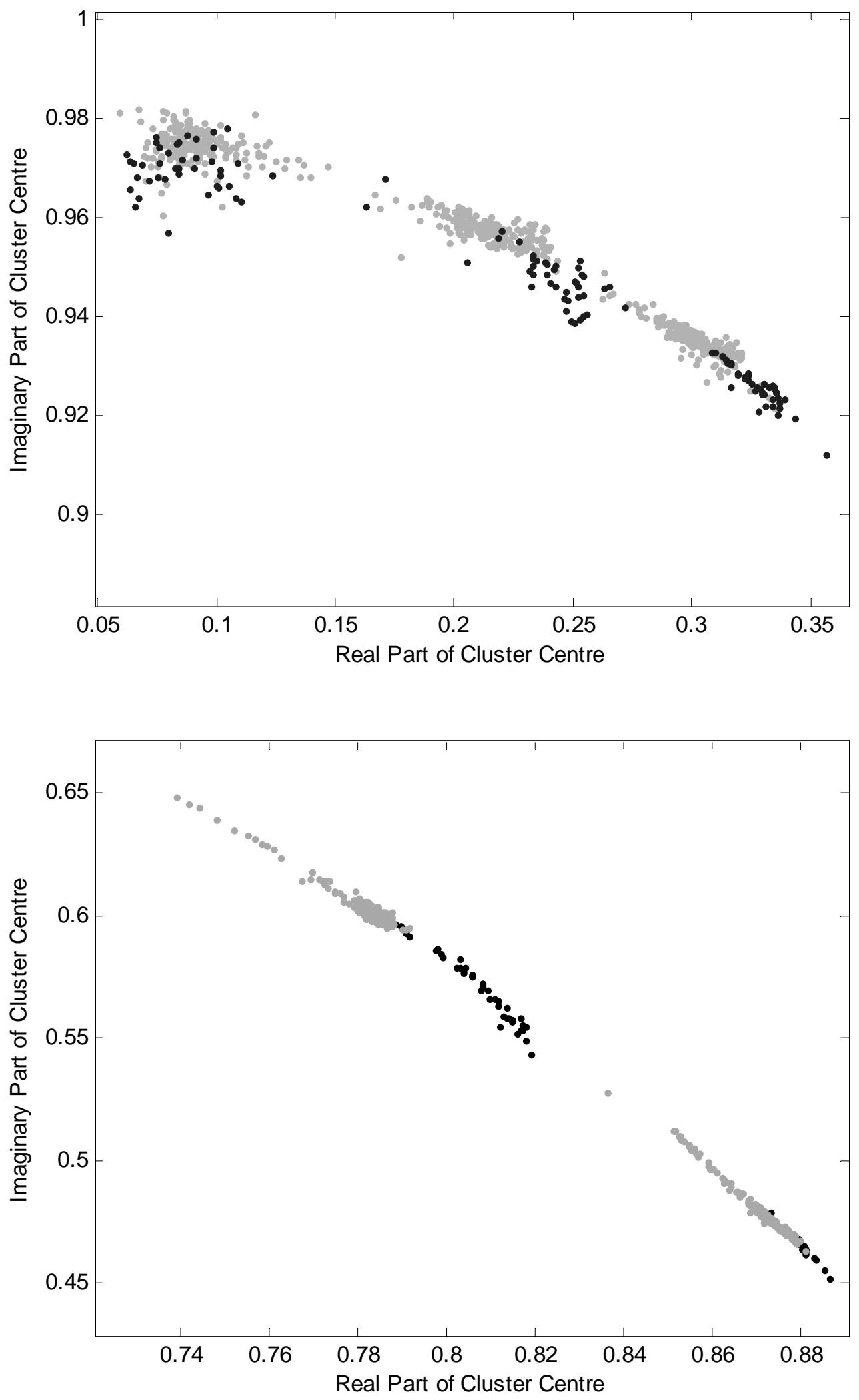

Figure 7. Clusters formed from analysis of Z24 Bridge data. The gray dots are cluster centres extracted from data prior to damage and the black dots are the cluster centres extracted after damage. (a) Frequency band of $9.3 \mathrm{~Hz}$ to $12.2 \mathrm{~Hz}$. This band contained two modes of the bridge. (b) Frequency band of $3.5 \mathrm{~Hz}$ to $6 \mathrm{~Hz}$. This band also contains two modes of the bridge. 


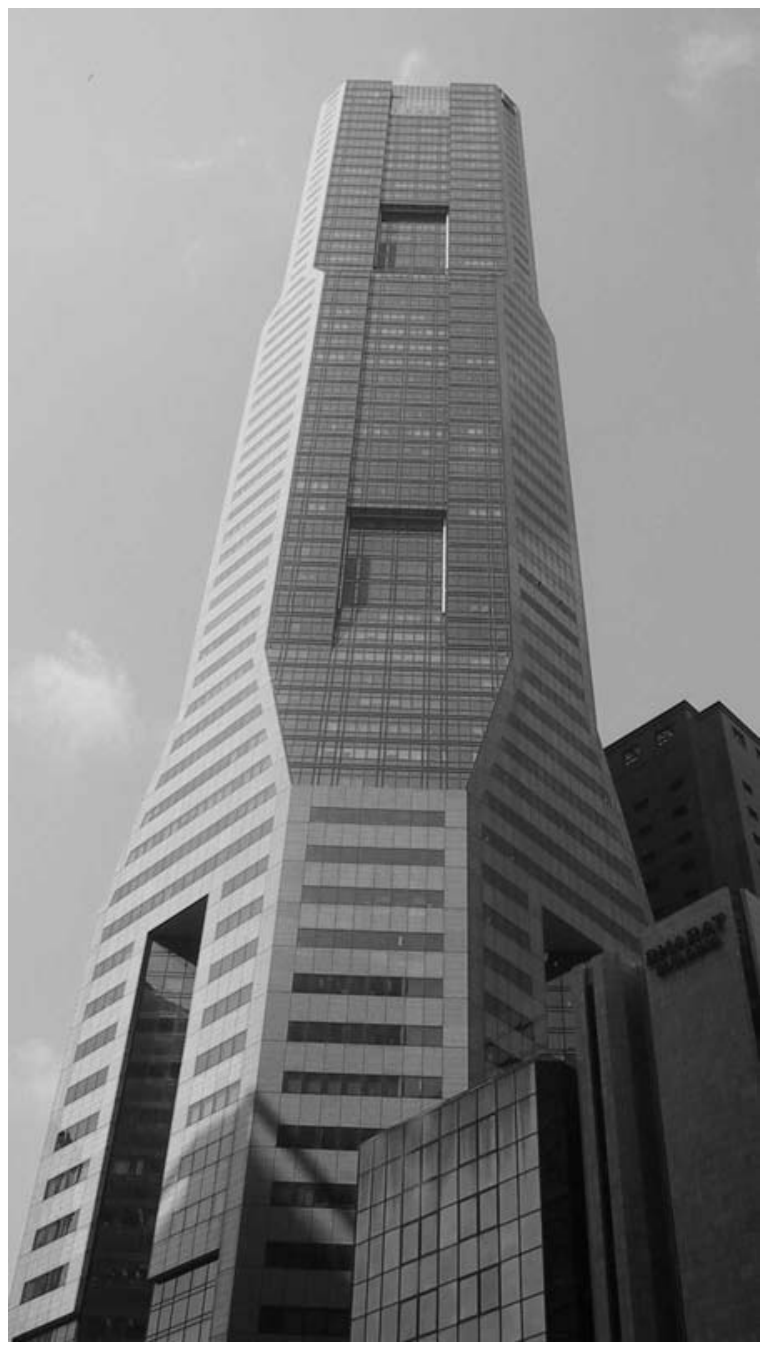

Figure 8. Republic Plaza Office Tower 


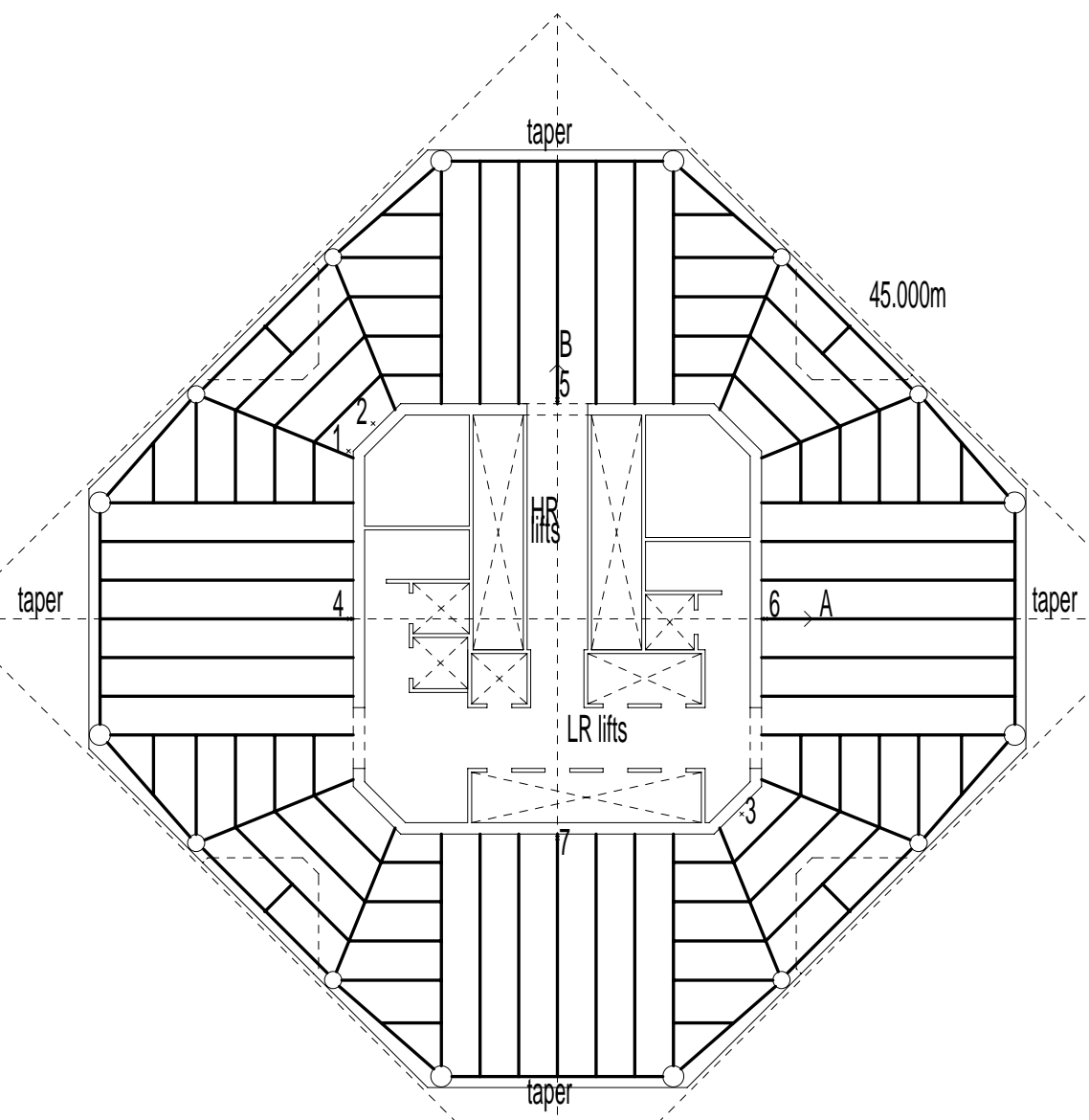

Figure 9. Section of Republic Plaza with axes A and B marked. 


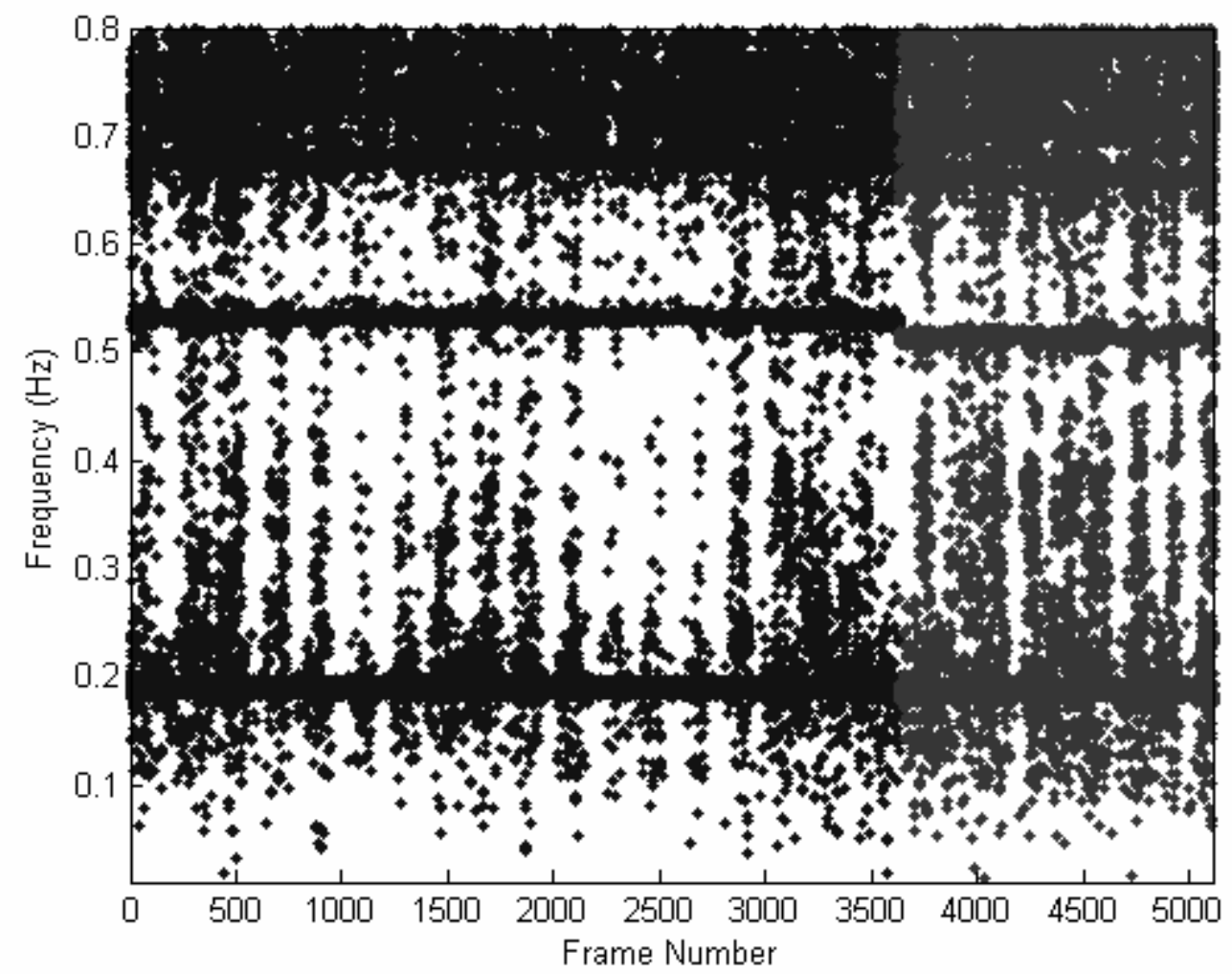

Figure 10a

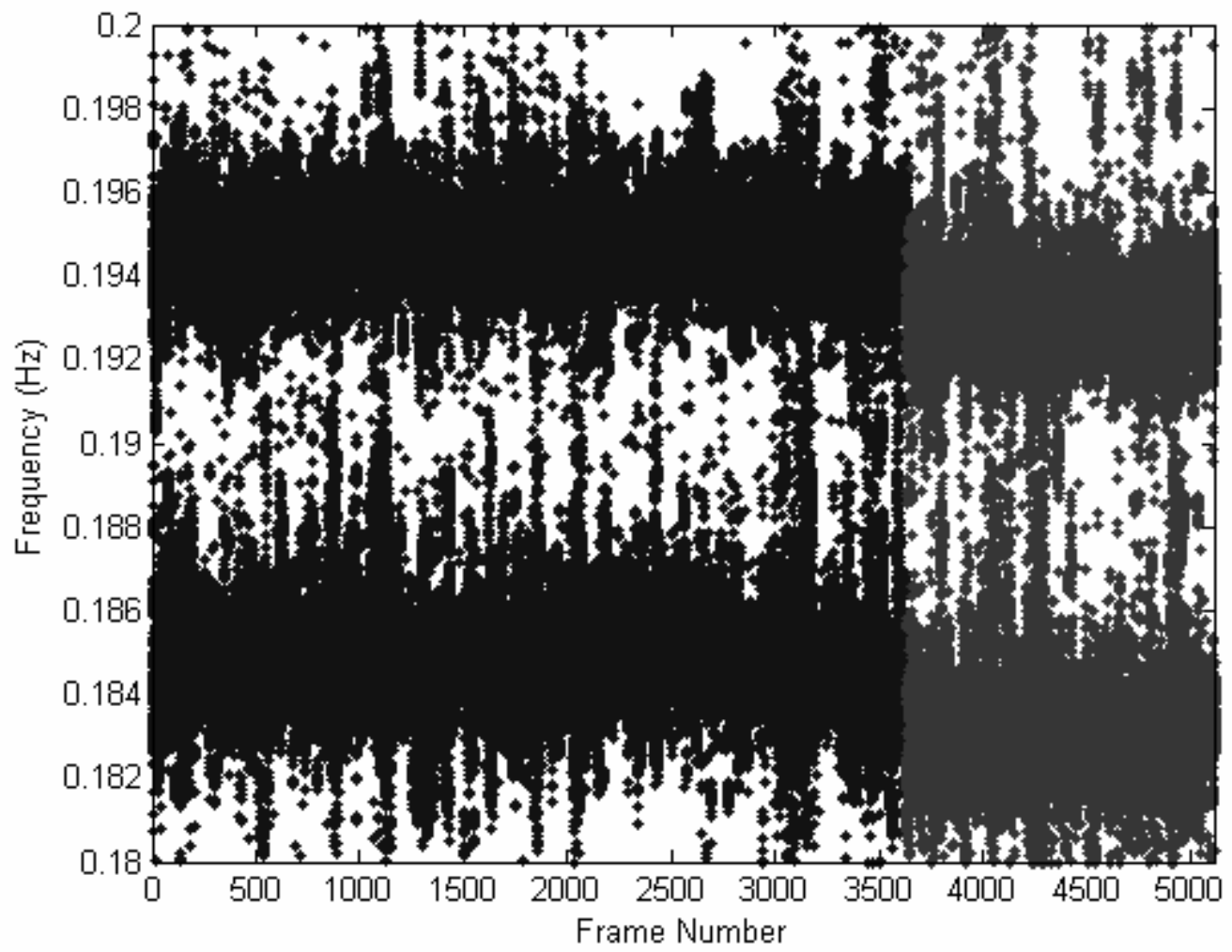

Figure $10 b$ 


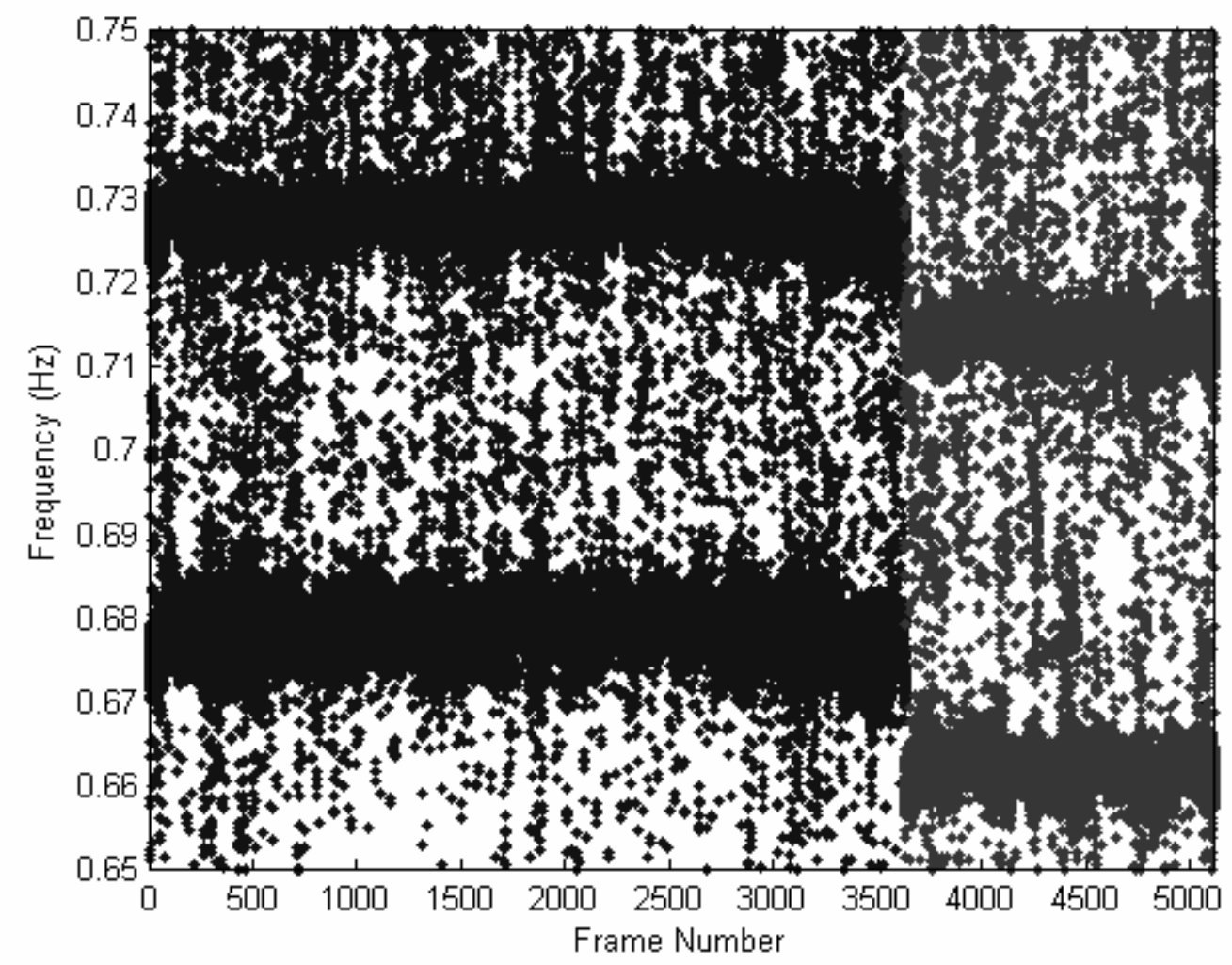

Figure 10c

Figure 10. (a) The stable poles extracted using SSI from two sets of response data from the Republic Plaza. (b) a zoom in on (a) to show the first two modes. (c) a zoom in on (a) to show the fourth and fifth modes. 


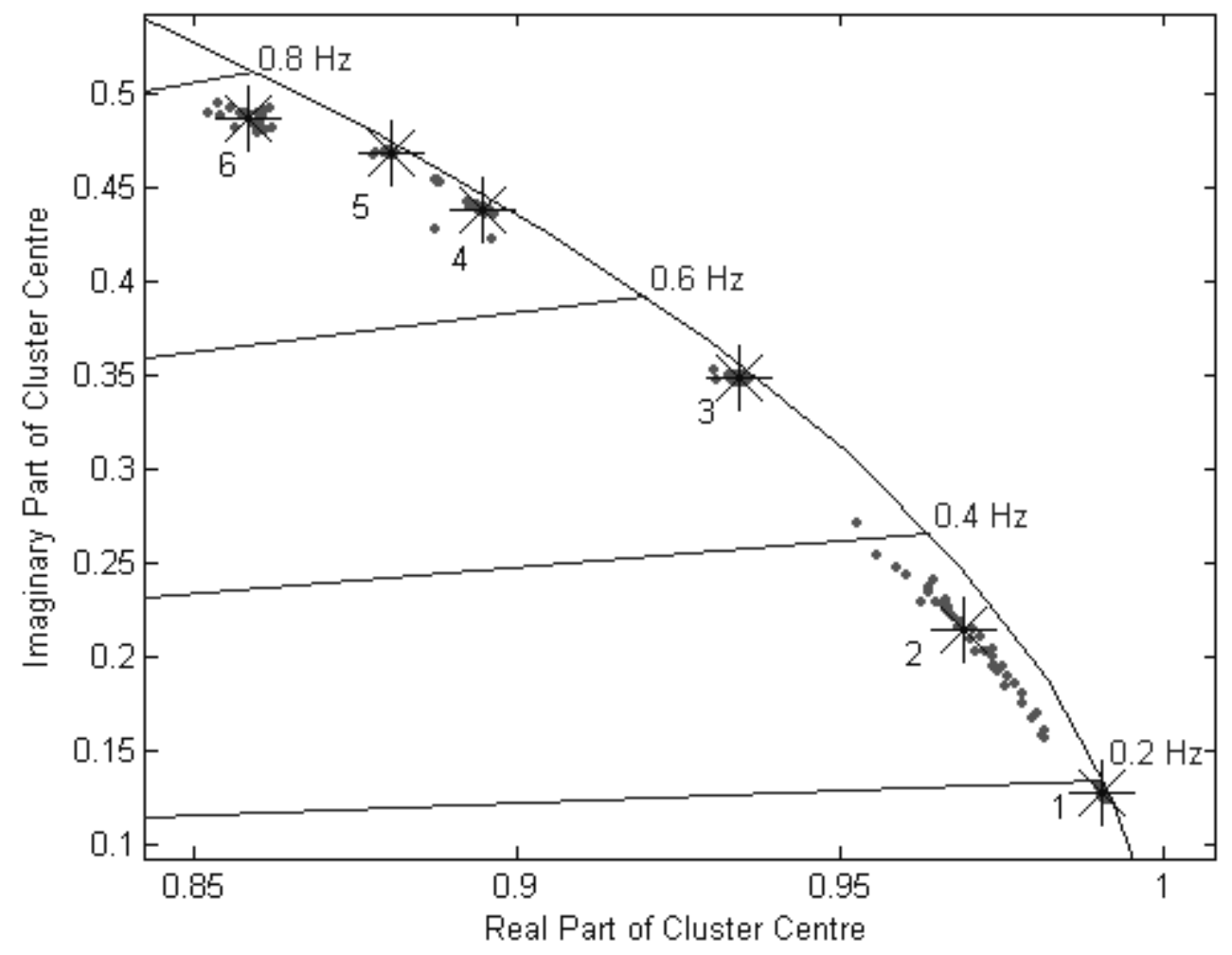

Figure 11. Clusters of centres formed in the Z-plane by clustering stable poles extracted from dataset RP1. Grey dots mark the cluster centres formed from the stable poles. Black stars mark the centres of the clusters formed by the dots. 


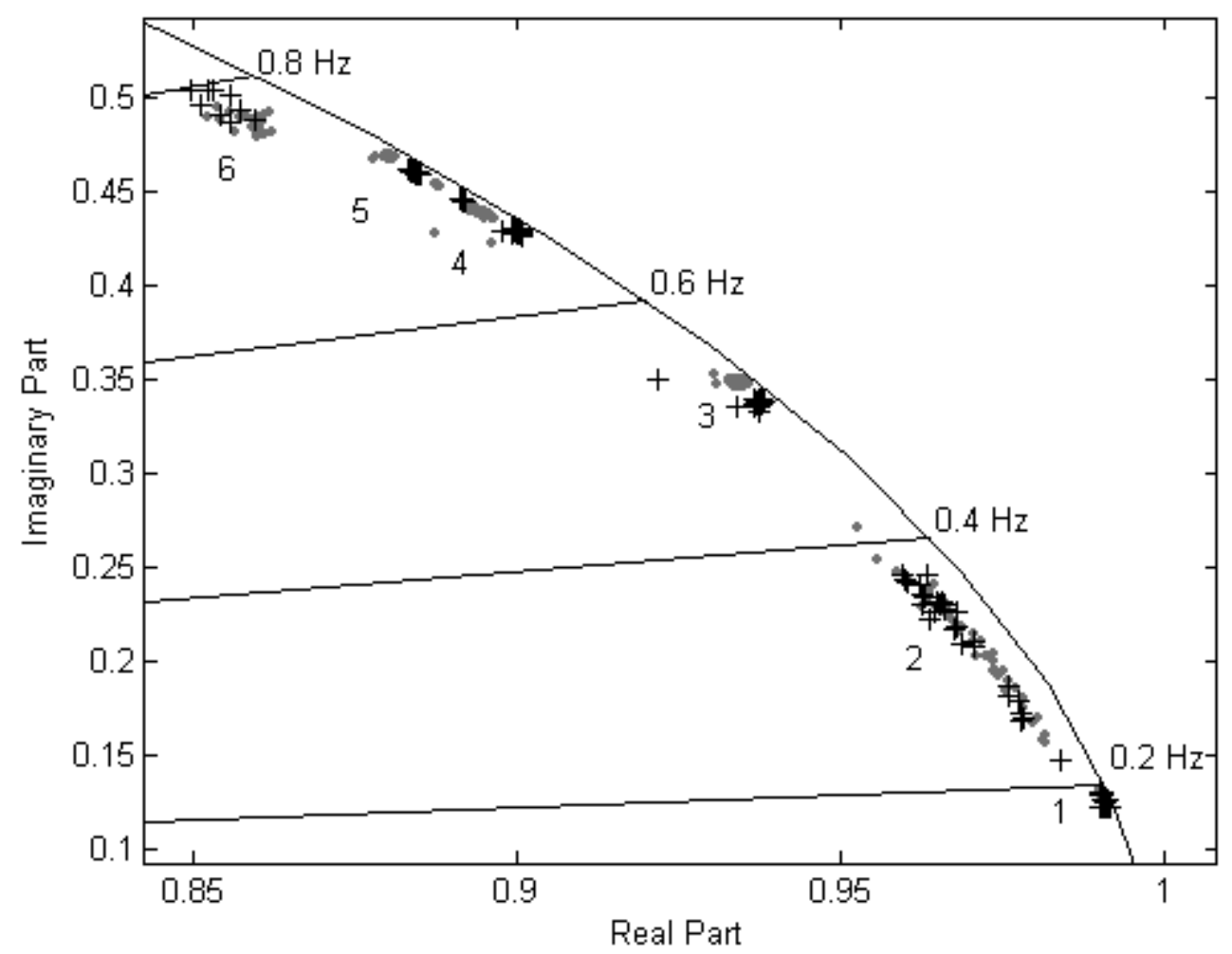

Figure 12. A comparison of the cluster centres extracted from RP1 (grey '.') and RP2 (black '+') 


\begin{tabular}{|l|c|l|c|}
\hline Cluster & Cluster Centre (Hz) & Membership (\%) & Compactness \\
\hline 1 & 0.19 & 27 & 0.0031 \\
\hline 2 & 0.32 & 6 & 0.0197 \\
\hline 3 & 0.53 & 22 & 0.0008 \\
\hline 4 & 0.68 & 22 & 0.0014 \\
\hline 5 & 0.73 & 20 & 0.0009 \\
\hline 6 & 0.77 & 3 & 0.0048 \\
\hline
\end{tabular}

Table 1. A comparison of membership and compaction of the six clusters seen in Figure 9. 